OPEN ACCESS

UWS Academic Portal

\title{
Caregivers' perception of dignity in teenagers with autism spectrum disorder
}

Mohammadi, Fatemeh; Rakhshan, Mahnaz; Molazem, Zahra; Zareh, Najaf; Gillespie, Mark

Published in:

Nursing Ethics

DOI:

$10.1177 / 0969733018796679$

Published: 01/11/2019

Document Version

Peer reviewed version

Link to publication on the UWS Academic Portal

Citation for published version (APA):

Mohammadi, F., Rakhshan, M., Molazem, Z., Zareh, N., \& Gillespie, M. (2019). Caregivers' perception of dignity in teenagers with autism spectrum disorder. Nursing Ethics, 26(7-8), 2035-2046.

https://doi.org/10.1177/0969733018796679

\section{General rights}

Copyright and moral rights for the publications made accessible in the UWS Academic Portal are retained by the authors and/or other copyright owners and it is a condition of accessing publications that users recognise and abide by the legal requirements associated with these rights. 
Reprinted by permission of SAGE Publications.

Mohammadi et al, Caregivers' perception of dignity in teenagers with autism spectrum disorder, Nursing Ethics, Volume number 26, Issue number 7-8, pp. 2035-2046. Copyright 2019 Sage Publications. DOI: https://doi.org/10.1177/0969733018796679 


\title{
Caregivers’ Perception of Dignity in Teenagers with Autism
}

\author{
Spectrum Disorder
}

\begin{abstract}
Introduction: Respecting the dignity of patients is one of the main ethical responsibilities of caregivers. However, in many cases, the dignity of patients, especially autistic teenagers is not maintained. The extent to which dignity needs are met for this group within the Iranian care system is difficult to determine as dignity is an abstract concept, and there are few related research studies reported.
\end{abstract}

Objectives: The objective of the present study is to find out what dignity means for autistic teenagers from the perspective of their caregivers.

Research design: This study uses a qualitative research design. The data was collected through individual, semi-structured interviews and field notes developed during the interviews. In order to analyze the data qualitative conventional content analysis was used.

Participants and research context: Sixteen professional caregivers for autistic teenagers working in public hospitals were recruited based on a targeted sampling method to reach data saturation from February 2016 to July 2017.

Findings: The findings of this study were presented in three main themes, "privacy”, "respecting individual identity” and “comprehensive support”, and 11 categories.

Ethical consideration: This study's protocol was approved by the Research Ethics Committee of medical universities located in Southeast of Iran and the required ethical principles were followed throughout.

Discussions and conclusion: Based on the findings of the present study from the perspective of caregivers, autistic teenagers need to be cared for and educated in a respectful environment where their privacy is maintained, their individual identities are respected and they receive comprehensive familial, social and financial support. These conditions would maintain the dignity of such teenagers and would result in appropriate behavioral outcomes. Therefore, it is suggested that a cultural, professional and institutional background in which all components of the autistic teenager's dignity are protected and emphasized be provided

Keywords: Dignity, autism, teenager, qualitative research 


\section{Introduction}

Maintaining the dignity is one of the fundamental human rights ${ }^{1}$. The term dignity has a lot of meanings, the most important of which are: value, honor, humanity, status, and respect ${ }^{2}$. The word "dignity" has increasingly become a part of contemporary discussion on health care ${ }^{3}$. Because an illness can create a background in which a person's dignity might be violated, all patients need to have their dignity maintained, even under adverse circumstances ${ }^{4,5}$. Therefore, maintaining and respecting the patients' dignity is one of the main rights of patients receiving healthcare ${ }^{6}$, and is also one of the moral responsibilities of caregivers ${ }^{7}$. The WHO states that all patients are entitled to receive treatment which maintains their dignity ${ }^{8}$. Therefore, many national and international nursing organizations have considered dignity of patients as one of the basics of caregiving ${ }^{9,10}$.

Respecting a person's dignity results in an increase in their satisfaction with care provided, improves the relationship between the caregiver and the patient, and also enhances the caregivers' motivations in providing their care ${ }^{11}$. However, some studies have mentioned that patients are vulnerable to losing their dignity within caregiving environments ${ }^{12,13}$ and some of the recent studies have reported a lack of privacy, respect and dignity within such environments ${ }^{14}$. Maintaining patient dignity is a crucial factor in guaranteeing high quality care delivery; therefore, understanding the concept of dignity from the perspectives of the patients or the caregivers makes the treatment process easier and enhances the psychological safety of the patients. Sufficient research is therefore required in order to fully explain the concept12 14 . Based on available studies, dignity is a complicated and multidimensional concept and therefore cannot have a simple definition ${ }^{13}$. Despite attempts to define this concept and identify its effective elements, the concept of dignity and its related factors remain complicated and unclear ${ }^{15}$. Since maintaining dignity cannot be confirmed without the concept being clearly defined ${ }^{16}$, a need has been recognized to define this concept and to identify the effective elements of dignity among patients with different diseases and disorders ${ }^{13}$.

People with intellectual and developmental disabilities, especially autistic children and teenagers, are more vulnerable to being ignored, abused, and not having their dignity maintained within caregiving environments ${ }^{17,18}$ Autism is one of the developmental disorders and according to recent research, this disorder has increased more than $6 \%$ throughout the world, creating significant challenges for caregiving organizations ${ }^{19}$.

Review of the related literature shows that dignity has been examined in specific categories of patients such as: cardiovascular patients ${ }^{20}$, end of life-stage patients ${ }^{21}$ as well as hospitalized teenagers ${ }^{22}$. However, this concept has not been defined for autistic teenagers. Due to their developmental and behavioral disorders, this group of patients are more vulnerable to be ignored and lose their dignity when in formal caregiving environments. Also, the results of the studies on other patients cannot be generalized to patients with intellectual, developmental or behavioral disorders, because the physical environment, organizational culture and the behaviors and perspectives of the care staff can affect dignity ${ }^{23}$. Thus, studies conducted among other patient groups cannot be generalized to autistic teenagers cared for within residential settings. On the other hand, the possibility of examining the concept of dignity from the perspective of autistic teenagers is difficult and ethically concerning, due to their developmental, behavioral and language 
disabilities. The caregivers of autistic teenagers are the most important and closest people to these teenagers; they spend long periods of time with these teenagers and have the most information about them. Hence, the aim of the present study was to explore the concept of dignity in teenagers with autism spectrum disorder from the perspective of Iranian caregivers, with a view to preserving and improving these teenager's dignity within health systems, and to reflect the necessity of exploring dignity in public health care settings in Iranian society.

Qualitative studies can help explain a phenomenon in the cultural context of people's perspectives who deal with a phenomenon for a long time ${ }^{24}$, so this approach was selected in order to explain caregivers perspectives of dignity among autistic teenagers. It is hoped that the results of this study can help managers and caregivers provide an environment which supports dignity and the rights of teenagers.

\section{Objectives}

The aim of the study is to explore the concept of dignity among autistic teenagers from the perspective of those caring for them.

\section{Methodology}

In this research, the authors are trying to explore dignity for looked after autistic teenagers from the perspective of their professional caregivers. Within qualitative research, reality is understood to be individually perceived, meaning a phenomenon can be understood through the commonalities and differences in how those closest to it make sense of $i^{24}$. Therefore, a qualitative approach with conventional content analysis has been used to investigate this subject. Conventional qualitative content analysis is an appropriate procedure for obtaining reliable and valid results from textual data allowing the creation of new knowledge and innovative understanding of phenomena under investigation. In qualitative content analysis, the raw data is based on inference and interpretations, then it is summarized and placed into categories and themes $25,26$.

Sixteen caregivers of autistic teenagers were interviewed, all of whom were working the in publicly funded centers providing care for autistic teenagers. These centers being affiliated to medical universities located in Southeast of Iran. Data gathering was performed from February 2016 to July 2017. The criterion for inclusion consisted of being Iranian, speaking and understanding Persian, being 24-55 years old, having at least two years of work experience in caregiving centers for autistic teenagers, and the ability to provide appropriate and sufficient data on the subject. The caregivers were selected through purposeful sampling method and were invited to participate in this study.

In this study, data collection was conducted through individual interviews; in so doing 16 in-depth semi-structured interviews were conducted with the 16 caregivers. The interviews were carried out face-to-face in quiet environments with the cooperation and willingness of the participants. In addition, field notes were also used allowing collection of what the interviewer sees, feels and experiences, as this helps with analysis of the concept. The individual interviews first started with general questions such as: "what does dignity mean for autistic teenagers? In which situations is 
the dignity of autistic teenagers at risk?” Based on the answers to these questions, follow-up questions such as "Can you explain more?" "What do you mean?" "Why did you feel so?” were asked. The interviews were carried out based on meeting the main objectives of this study. Also, if necessary, follow-up questions were used in order to increase and clarify information obtained, such as: "Can you explain more? Can you give me an example?" The interviews were recorded and field notes were taken with the permission and awareness of the caregivers. Each interview lasted between 45 and 90 minutes. Immediately after each interview, the interviews were listened to by the first author several times to develop a general understanding and deep insights, and then the interpretations (do you mean interviews?) were transcribed on paper. (Should this read “transcribed verbatim”) Data analysis was carried out after each interview, and later interviews were then scheduled. Interviews continued until the data was saturated. Saturation occurs when there is no new categories emerging and the categories are saturated based on their characteristics and dimensions ${ }^{27}$.

The data were analyzed simultaneously using the content analysis method; first each text was reviewed for immersion and acquiring insights and deep understanding around the phenomenon under study. Then meaning units were determined based on the objectives and the study questions. Next, important points were extracted as open codes, considering their clear and hidden meaning units. These codes were categorized under broader titles based on their similarities and differences, and the data analysis continued until the themes were extracted ${ }^{25,27 .}$

In order to ensure trustworthiness Graneheim and Lundman's criterion were used ${ }^{28}$. At the beginning of the study, the researchers bracketed all their prior information and personal beliefs regarding maintaining dignity in the care setting I order to avoid the influence and interference of personal beliefs in the investigation of this phenomenon. To increase the reliability and validity of findings several techniques were employed including combining review of data sources (semistructured interviews and field notes), prolonged engagement with the data, member checking, and peer checking. To do this, the extracted concepts and themes were submitted to 4 participants and 2 peers; who stated that the findings were in line with their understandings and interpretations. Furthermore, the researcher limited the textual reviews in order to reduce bias in collecting, analyzing and coding of the interviews to increase the validity of the date. Finally, Confirmability was acquired through exact recording of participant narratives and detailed reporting of the study to provide the possibility of follow-up for other researchers.

\section{Ethical Considerations}

The institutional review board of the medical universities located in Southeast of Iran provided ethics approval (approval number: 95-01-08-1168). Also, at the beginning of each interview, the researcher introduced herself and explained the aims of this study, and informed consent was obtained after providing verbal and written explanations. The participants were assured that all information would remain confidential. The researcher created the opportunity for participants to inform the researcher about their withdrawal from the study at any stage of the study and assured them that their lack of participation or withdrawal would not have any consequences for them. 


\section{Results}

159

160

161

162

163

164

165

166

167

168

169

170

171

172

173

174

175

176

177

178

179

180

181

182

183

184

185

186

187

188

In this study interviews with 16 caregivers of autistic teenagers living in public health centers were carried out. The participants of this study included 10 women and 6 men. The individual characteristics of the participants are presented in Table 1.

The three main themes emerging from these interviews were privacy, respecting individual identity, and comprehensive support while 11 categories were extracted from the data. Table 2 presents the themes and categories. Dignity for teenagers with autism spectrum disorder is consequently defined as the preservation of privacy; respect for individual identity and the delivery of comprehensive support for these teenagers.

\section{A) Privacy}

The participants in this study stated that maintaining the sexual, physical, psychological and information privacy of autistic teenagers receiving care in in publicly funded care centers is very important in order to maintain their dignity. therefore, the caregivers respect the patients' privacy and try to maintain it. The privacy theme included four categories: maintaining physical-sexual privacy, maintaining psychological privacy, maintaining information privacy, and respecting the possessive rights of autistic teenagers.

The participants in this study reported that due to the patients developmental, behavioral and intellectual disorders, and the high prevalence of reports of physical and sexual abuse of such teenagers, it is essential that they be taken care of by same-sex caregivers.

"Frequently, when delivering care, some parts of the autistic teenagers' bodies, especially their sexual organs might be exposed and obviously. based on these teenagers' disorders if the caregiver is the same-sex, it would be safer" (Female, 42 years old).

They also said that due to behavioral disorders, such teenagers might show abnormal behaviors which might threaten the physical or sexual privacy of themselves and other people. Therefore, it is essential that autistic teenagers of the same-sex reside together within shared accommodation.

"Sometimes these teenagers show certain behaviors: they take off their clothes in their room in the health center; thus, they may threaten the physical and sexual privacy of themselves or their roommates. So keeping teenagers of the same sex in one room can help support their privacy." (Male, 38 years old). 
The caregivers insisted that male teenager should be wearing his pants (trousers) to maintain his sexual and physical privacy. The researcher overheard the following conversation in a center providing care for autistic teenagers:

Caregiver: Put on your pants immediately.

Teenager with aggression: I will not

Caregiver: If you wear pants, you can play with others

He subsequently wears pants with the help of a caregiver

Caregivers emphasized that care should be delivered by staff of the same gender as the patient, in order to maintain their sexual and physical privacy. The researcher overheard the following within a center providing care for autistic teenagers:

Supervisor: How many adolescents are hospitalized ?

Caregiver: $\quad 6$ boys and 4 girls. That means we need 3 male caregivers and 2 female caregivers.

Supervisor: $\quad$ ok

Some of the caregivers also mentioned that it is important to respect the psychological privacy of these teenagers; when teaching appropriate behaviors, we should pay attention to their developmental status and not use their disabilities in a way that destroys their personality or selfesteem.

"Caring and educating these teenagers is difficult. Sometimes the caregivers ignore their developmental and physical disorders while educating them, and when these teenagers get aggressive and quarrelsome, they will be stigmatized with inappropriate labels; even mentioning their disabilities can really ruin their self-esteem" (Female, 30 years old).

In addition, the participants in this study stated that the personal information and the personal lives of these teenagers and especially history of sexual rape or abuse should not be recorded in their files, but should be rather kept confidential by the head nurse or the doctor of the caregiving center.

"Although it is important to keep all the teenagers' information confidential, it is apparent that in our country there is a negative view toward abuse, especially sexual assault, and sometimes the file might be studied by people other than the medical staff; therefore, it is essential that such information be kept confidential by the person in charge" (male, 28 years old).

The participants in this study also said that the caregivers should respect the rights of these teenagers around use of their personal items and accessories.

"Although these teenagers have developmental and behavioral disabilities, the caregivers should respect them and understand that they do not have the right to take one of the teenagers' posessions and give it to another teenager without their permission. 
Sometimes the caregivers do this, which results in arguments or fights among teenagers or arguments between the teenager and the caregiver" (male, 40 years old).

\section{B) Respecting individual identity}

The caregivers who participated in the study stated that it is an ethical principle to respect religious and age identity of autistic teenagers. Also, the caregivers should avoid pity and discrimination in order to maintain the dignity of these teenagers, and should care for such teenagers fairly. This theme included the four categories of respecting age identity and religious identity of autistic children, avoiding pity and discriminatiory behaviors.

One of the moral principles of nursing care is respecting the age and religious identities of the people receiving care in order to provide appropriate care.

"These are not kids. These teenagers have good physical development and they have grown up and we should respect their maturity and age" (Female, 28 years old)

"These teenagers possess different religious beliefs and I, as a caregiver, respect the identity and religious needs of these teenagers and their families, and respect what they say even if I dont agree with their beliefs" (Female, 28 years old).

Caregivers also suggested that it is essential to avoid any unnecessary pity in order to promote patient dignity.

"It's not appropriate to demonstrate unnecessary pity while educating and caring for these teenagers, working over sympathetically and being guided by extensive levels of unnecessary compassion can destroy their personality and makes us unable to perform our duty to educate them appropriately" (male, 38 years old).

In addition, it is vital that the caregivers avoid demonstrating any discrimination when caring for these teenagers and even parents should avoid any discrimination between autistic teenagers and other teenagers in the family and approach them each fairly.

"Sometimes families differentiate between autistic teenagers and their siblings and pay less attention to these teenagers and their needs and pay more their attention to their fit and well teenager and therefore are discriminating toward their children" (male, 30 years old).

\section{C) Comprehensive support}

Based on the statements of the participants, comprehensive support for autistic teenagers is one of the most important dimensions for creating and promoting dignity for this client group, therefore, it is necessary that these teenagers be provided with comprehensive 
251

252

253

254

255

256

257

258

259

260

261

262

263

264

265

266

267

268

269

270

271

272

273

274

275

276

277

278

279

280

281

282

283

284

285

286

287

288

familial, social and financial support. This theme includes three categories: familial support, financial support and Social awareness support.

The participants in this study stated that familial support and especially the respect that the parents consider for such teenagers is the most effective factor promoting their dignity.

"The parents of some autistic teenagers are so respectful toward their teenager and they are so patient toward the teenager's aggressive behavior, also important is appropriate training and education, which unconsciously makes all caregivers and even the relatives of these teenager more likely to behave politely with them. I think the support and demonstration of respectful behavior by the family with these teenagers has the biggest effect on maintaining dignity of the teenagers by others" (female, 41 years old).

Furthermore, the participants reported that if people in general are aware of the persons illness and know that the inappropriate behavior of these teenagers is due to that, they are likely to have more appropriate reactions toward the teenager's behavior and therefore their dignity will be maintained within that society.

"These teenagers will demonstrate a lot of inappropriate behavior in public and crowded places and because of the lack of awareness about their disorder; people would chastise autistic teenagers and their caregivers verbally and make them feel uncomfortable, alienated and sad. Therefore, if public awareness is increased, people are likely to respond moree helpfully in reaction to such inappropriate behavior, maintaining the patients dignity" (Female, 32 years old).

The participants also added that caring for autistic teenagers attracts a significant financial burden for families. As a result, there is a lot of ignorance in educating and caring for such teenagers and this will violate the dignity of such teenagers at present and in future; so it is needed to have comprehensive financial support of these teenagers by their families or public organizations in order to promote their dignity.

"Autistic teenagers need to be educated for a long time. Because without extensive education, their social skills will not improve and they won't be able to live independently; consequently, their dignity will be damaged. On the other hand, providing prolonged education will require large sums of funding. Hence, financial support for these teenagers by governmental organizations is needed" (male, 27 years).

\section{Discussion}

Human dignity is an important concept in nursing and professional caregiving, and it is one of the fundamental human rights ${ }^{29}$. Since teenage patients are more vulnerable, their dignity tends to be more at risk, compared to other groups of patients. Therefore, because of their behavioral and developmental disabilities, can be claimed that dignity of autistic teenagers is more at stake 
compared to other hospitalized teenagers, the findings of this study emphasizes the importance of maintaining dignity among autistic teenagers. In this study, maintaining dignity of autistic teenagers from the perspectives of caregivers included three main themes: privacy, respecting individual identity, and comprehensive support.

The concept of dignity in autistic adolescents has not been explored and defined, Therefore, Due to the lack of relevant studies the findings of this study were discussed with the findings of others studies that were explored the concept of dignity on other patients' population.

Privacy is fundamental to human dignity; it is essential to respect the patient's privacy, especially hospitalized teenagers. The present study, based on the perspectives of caregivers for autistic teenagers, revealed that maintaining the physical-sexual, psychological, informational privacy and respecting the possessive rights of teenagers suffering from autism is of great importance. The human dignity is a concept related to the culture, also all caregivers of the present study were Muslims and Iranians and they believe that hospitalized teenagers should also respect the Islamic laws. Furthermore, these caregivers knew that physical-sexual issues were of great importance in Iranian culture and especially rape threatened the personal and familial dignity. Therefore, caregivers tried to keep teenagers of the same sex in one room and also provide professional care by a person of the same sex in order to maintain their dignity. Three related studies also revealed that maintaining physical-sexual privacy among hospitalized adolescents is very important in order to maintain their dignity22, 30, 31. In accordance with the findings of the present study, caregivers should respect the psychological privacy of these teenagers; especially when teaching correct behaviors to these teenagers. A lot of related studies have examined psychological and psychological abuse toward children and teenagers with developmental and intellectual disorders such as autism ${ }^{32-34}$. In this regards, Reiter's study reports that children and teenagers suffering from developmental and mental disorders are very vulnerable toward stigma, ignorance and psychological abuses ${ }^{35}$. Caregivers of the present study stated that personal, clinical, and familial information of autistic teenagers should be quite confidential especially the information related to the teenager's sexual abuse should be kept in locked file by head nurse, and should be given to the medical staff only if necessary. One study in Iran on hospitalized teenagers reported that maintaining the information privacy of hospitalized teenagers from their perspective is one of the most important aspects of privacy; these teenagers share their information only with their nurses and avoid revealing their personal information to other people, which reveals the importance of this aspect in maintaining the privacy of patients ${ }^{22}$. Respecting the possessive rights of autistic teenagers is another category related to the theme of privacy in the present study. The caregivers in the present study stated that although due to behavioral and developmental disorders the autistic teenagers are unable to participate in caregiving decisions, and such decisions are mainly made by their families and the medical staff, still their possessive rights about their bodies and personal items should be respected and their bodies and personal items should not be touched without their permission. Respecting the possessive rights was referred to as protection of autonomy in other studies; this difference can be due to the developmental-behavioral disabilities of autistic teenagers and the lack of their ability in order to participate in medical decision-making. Therefore, maintaining the physical-sexual, psychological, informational privacy 
and respecting the possessive rights of these teenagers are necessary for improve the quality of care.

Respecting the personal identity is another theme of dignity among autistic teenagers in the present study. Respecting the individual identity in the present study emphasizes respecting the age and religious identity, avoiding pity and discrimination behaviors in order to develop the dignity among autistic teenagers. The participants of this study believed that they needed to respect the age identity and different religious beliefs of autistic teenagers and their families, and should try to meet the religious and spiritual needs of such teenagers. A number of related studies mention the importance of respecting the personal identity by the medical staff as one of the important aspects of ethical care 36, 37, Therefore nurses and other caregivers should respect the patients' national, religious, racial and age differences, and should provide the care without any discrimination ${ }^{38} 39,40$. Furthermore, the caregivers of this study reported that pity behavior toward these teenagers should be avoided, because such behavior can interfere with the education and caregiving and consequently destroy the self-esteem of these teenagers and threaten the dignity of autistic teenagers. Other studies also stated pity toward patients can have adverse effects on the patients' psychological attitudes and their dignity ${ }^{41-43}$. Avoiding discrimination is also another important category related to the theme of respecting personal identity. Participants of this study stated that caregivers should not differentiate between autistic teenagers; even the parents of such children should not differentiate between autistic children and fine children, because it results in threatening the dignity of autistic teenagers. Also avoiding discrimination is a professional value in nursing; caregivers should pay attention not to have discrimination between patients. two other studies also state that there are a lot of evidences that caregivers differentiate between patients and this ethical values are ignored by caregivers 44,45 .

Another theme in this study is comprehensive support of autistic teenagers. The caregivers who participated reported the comprehensive support of the autistic teenagers is one of the main needs of such teenagers in order to improve their dignity. These teenagers needed comprehensive familial support in their physical and psychological aspects; social awareness support and financial support. A lot of studies have examined the importance of social and familial support of adolescents; all of them state that such support will enhance the teenager's ability to cope with present situation and improve teenager's behavior ${ }^{46}$. However, most studies among autistic children and teenagers have examined the importance and effects of familial support and social support of the parents as main caregivers of such children and teenagers ${ }^{47,48}$. Bilgin et al. state that professional and social support will reduce the parents' worries and fears about their autistic children and teenagers ${ }^{49}$. It is obvious that if the parents of such children and teenagers have the familial and social support, they will be more competent in order to care for their disabled children and teenagers. Also they can better maintain and improve the dignity of teenagers suffering from developmental and intellectual disabilities. Furthermore, the participants of this study added that caring for such children imposes a lot of expenses; thus financial support of public centers is important. Although, many developed countries have centers and communities for the financial support of disabled children and teenagers. But in most 
developing countries, especially Iran, there are not public centers which support these teenagers and their families financially due to the adverse economic situations.

Finally, it can be said that one of the most important care needs of autistic teenagers is to preserve their dignity. The caregivers participating in the present study felt the dignity of these teenagers has been preserved when they were cared in a respectful atmosphere with preservation of their privacy and had also receive the comprehensive familial, social and financial support.

\section{Limitations and suggestions}

One of the limitations of the present study was that the caregivers of the autistic teenagers were only from public centers. The selection of the participants from private centers could improve the generalizability of the findings. Another limitation of this study was the collection of data through individual interviews and field notes; making use of other data collection methods could enrich the results of this qualitative study. Therefore, it is suggested that futures studies examine the dignity of autistic teenagers and teenagers with other developmental or behavioral disorders in private centers also in addition to individual interviews, other data collection methods such as observations and focus groups be utilized. Also, more qualitative and quantitative research on larger samples in other places and cultures is needed to explore the concept of dignity in autistic adolescents.

\section{Conclusion}

Autistic teenagers are more vulnerable to losing their dignity. Based on the fact that preserving dignity in teenagers can have desirable outcomes, dignity is of great importance, but dignity has not been defined in autistic teenagers. Findings of this study help to define this concept in this grope of patients. According to the results of the present study, providing a supportive atmosphere while respecting the autistic teenager's privacy and paying attention to their individual identity as well as can help to preserve their dignity. In addition, more emphasis on maintaining physical-sexual privacy and respecting the possessive rights of autistic teenagers along with comprehensive support in care helps improve the quality of care and promote of dignity in these teenagers.

Since human dignity is an essential value of nursing, especially in autistic adolescents. Therefore it is essential to provide the cultural, professional and organizational environment in which all the aspects of dignity of autistic teenagers are respected. Findings of this study can lead to pay attention to autistic teenager's dignity in nursing education in Iran, and Pediatric nurse specialist respect the dignity of these teenagers and are sensitive to this important issue. In addition the authorities and policy makers of health organizations should examine and use the findings of this study in order to provide appropriate environments regarding the dignity of these teenage in different aspects.

\section{Conflict of interest}

No conflict of interest was reported by the authors.

\section{Acknowledgment}


The present study is a result of the research project issued by medical universities located in Southeast of Iran, therefore the researchers wish to express their gratitude toward the authorities of Nursing and Midwifery Departments, and they also like to further thank the caregivers who participated in this study and other people who helped them carry out this research.

(1)

6

(1)

(1)

\section{References:}

1. Michael L. Defining dignity and its place in human rights. The new bioethics. 2014; 20: 12-34.

2. Dehkhoda AA. Dehkhoda Dictionary (Vol. 8). Tehran, Dehkhoda Dictionary Institute. 1994.

3. Bagheri $h$, yaghmaei f and ashktorab t. Patient dignity and related factors: a qualitative metasynthesis study. Journal of Knowledge \& Health. 2011; 6.

4. Gustafsson L-K, Wigerblad $\AA$ and Lindwall L. Undignified care: Violation of patient dignity in involuntary psychiatric hospital care from a nurse's perspective. Nursing ethics. 2014; $21: 176-86$.

5. Whitehead J and Wheeler H. Patients' experiences of privacy and dignity. Part 1: a literature review. British Journal of Nursing. 2008; 17: 381-5.

6. Berglund B, Anne-Cathrine $M$ and Randers I. Dignity not fully upheld when seeking health care: Experiences expressed by individuals suffering from Ehlers-Danlos syndrome. Disability and rehabilitation. 2010; 32: 1-7.

7. Sizoo EM, Taphoorn MJ, Uitdehaag B, et al. The end-of-life phase of high-grade glioma patients: dying with dignity? The oncologist. 2013; 18: 198-203.

8. Organization WH. A declaration on the promotion of patients' rights in Europe. Copenhagen: WHO Regional Office for Europe. 1994.

9. Association AN. Code of ethics for nurses with interpretive statements. Nursesbooks. org, 2001. 10. Zahedi F, Sanjari M, Aala M, et al. The code of ethics for nurses. Iranian journal of public health. 2013; $42: 1$.

11. Sadeghi T and Dehghan Nayyeri N. Patients' dignity: patients' and nurses' perspectives. Iranian Journal of Medical Ethics and History of Medicine. 2009; 3: 9-20.

12. Ebrahimi H, Torabizadeh C, Mohammadi E and Valizadeh S. Patients' perception of dignity in Iranian healthcare settings: a qualitative content analysis. Journal of medical ethics. 2012: medethics2011-100396.

13. Matiti MR and Trorey GM. Patients' expectations of the maintenance of their dignity. Journal of clinical nursing. 2008; 17: 2709-17. 
472

473

474

475

476

477

478

479

480

481

482

483

484

485

486

487

488

489

490

491

492

493

14. Gallagher A, Li S, Wainwright P, Jones IR and Lee D. Dignity in the care of older people-a review of the theoretical and empirical literature. BMC nursing. 2008; 7: 11.

15. Adib-Hajbaghery M and Aghajani M. Patients dignity in nursing. Nurs Midwifery Stud. 2015; 4: e22809.

16. Baillie L, Gallagher A and Wainwright P. Defending dignity: challenges and opportunities for nursing. Royal College of Nursing, 2008.

17. Mandell DS, Walrath CM, Manteuffel B, Sgro G and Pinto-Martin JA. The prevalence and correlates of abuse among children with autism served in comprehensive community-based mental health settings. Child abuse \& neglect. 2005; 29: 1359-72.

18. Slingsby B, Yatchmink $Y$ and Goldberg A. Typical skin injuries in children with autism spectrum disorder. Clinical pediatrics. 2017; 56: 942-6.

19. Smith LE, Hong J, Seltzer MM, Greenberg JS, Almeida DM and Bishop SL. Daily experiences among mothers of adolescents and adults with autism spectrum disorder. Journal of autism and developmental disorders. 2010; 40: 167-78.

20. Moraveji M, Yaghmaei F and Bagheri H. Dignity of heart failure patients referred to Zanjan hospitals. Journal of Health Promotion Management [Research]. 2015; 4: 75-83.

21. Hemati Z, Ashouri E, AllahBakhshian M, et al. Dying with dignity: a concept analysis. Journal of clinical nursing. 2016; 25: 1218-28.

22. Jamalimoghadam N, Yektatalab S, Momennasab M, Ebadi A and Zare N. Hospitalized adolescents' perception of dignity: A qualitative study. Nursing ethics. 2017: 0969733017720828.

23. Nursing RCo. Defending dignity: challenges and opportunities for nursing. Royal College of Nursing London, 2008.

24. LoBiondo-Wood G and Haber J. Nursing Research-E-Book: Methods and Critical Appraisal for Evidence-Based Practice. Elsevier Health Sciences, 2017.

25. Elo $\mathrm{S}$ and Kyngäs $\mathrm{H}$. The qualitative content analysis process. Journal of advanced nursing. 2008; 62: 107-15.

26. Zhang Y and Wildemuth BM. Qualitative analysis of content. Applications of social research methods to questions in information and library science. 2016; 318.

27. Speziale HS, Streubert HJ and Carpenter DR. Qualitative research in nursing: Advancing the humanistic imperative. Lippincott Williams \& Wilkins, 2011.

28. Graneheim UH and Lundman B. Qualitative content analysis in nursing research: concepts, procedures and measures to achieve trustworthiness. Nurse education today. 2004; 24: 105-12.

29. Hall P. Dignity and health. The Lancet. 2013; 382: 28.

30. Narayan K, Hooker C, Jarrett C and Bennett D. Exploring young people's dignity: A qualitative approach. Journal of paediatrics and child health. 2013; 49: 891-4.

31. Scott $\mathrm{P}$, Vlimki M, Leino-Kilpi $\mathrm{H}$, et al. Autonomy, privacy and informed consent 1: concepts and definitions. British journal of nursing. 2003; 12: 43-7.

32. Balogh R, Bretherton K, Whibley $S$, et al. Sexual abuse in children and adolescents with intellectual disability. Journal of Intellectual Disability Research. 2001; 45: 194-201.

33. Bowen E and Swift C. The Prevalence and Correlates of Partner Violence Used and Experienced by Adults With Intellectual Disabilities: A Systematic Review and Call to Action. Trauma, Violence, \& Abuse. 2017: 1524838017728707.

34. Monteiro ACdS, Fernandes ATRS, Oliveira ABMd, Peixoto IVP and Pamplona MCdCA. Mothers' perspective on violence against children: constructing meanings. Revista brasileira de enfermagem. 2018; 71: 34-9.

35. Reiter S, Bryen DN and Shachar I. Adolescents with intellectual disabilities as victims of abuse. Journal of Intellectual Disabilities. 2007; 11: 371-87. 
36. Jo K-H and Doorenbos AZ. Understanding the meaning of human dignity in Korea: a content analysis. International journal of palliative nursing. 2009; 15: 178-85.

37. Sletteb $\varnothing \AA$, Caspari S, Lohne V, Aasgaard T and Nåden D. Dignity in the life of people with head injuries. Journal of Advanced Nursing. 2009; 65: 2426-33.

38. Faulkner $\mathrm{J}$ and Laschinger $\mathrm{H}$. The effects of structural and psychological empowerment on perceived respect in acute care nurses. Journal of nursing management. 2008; 16: 214-21.

39. Büssing A and Koenig HG. Spiritual needs of patients with chronic diseases. Religions. 2010; 1: 18-27.

40. Kang K, Im J, Kim H, Kim S, Song M and Sim S. The effect of logotherapy on the suffering, finding meaning, and spiritual wellbeing of adolescents with terminal cancer. Journal ofCurrent Directions in Psychological Science. 2009; 312.

41. Jones J and Pattison S. Compassion as a philosophical and theological concept. Compassion in nursing: theory, evidence and practice London: Palgrave. 2016: 43-56.

42. Sinclair S, Beamer K, Hack TF, et al. Sympathy, empathy, and compassion: A grounded theory study of palliative care patients' understandings, experiences, and preferences. Palliative medicine. 2017; 31: 437-47.

43. Solomon RC. Sympathy and Vengeance: The Role of the Emotions in. Emotions: Essays on emotion theory. 2013: 291.

44. Papastavrou E, Efstathiou $G$ and Andreou C. Nursing students' perceptions of patient dignity. Nursing ethics. 2016; 23: 92-103.

45. Shafakhah M, Molazem Z, Khademi M and Sharif F. Facilitators and inhibitors in developing professional values in nursing students. Nursing ethics. 2018; 25: 153-64.

46. Pinkerton J and Dolan P. Family support, social capital, resilience and adolescent coping. Child \& family social work. 2007; 12: 219-28.

47. Joosten AV and Safe AP. Management strategies of mothers of school-age children with autism: Implications for practice. Australian occupational therapy journal. 2014; 61: 249-58.

48. Safe A, Joosten A and Molineux $M$. The experiences of mothers of children with autism: Managing multiple roles. Journal of Intellectual and Developmental Disability. 2012; 37: 294-302.

49. Bilgin $\mathrm{H}$ and Kucuk L. Raising an autistic child: Perspectives from Turkish mothers. Journal of Child and Adolescent Psychiatric Nursing. 2010; 23: 92-9.

(1)

(1)

(1)

8


540

541

542

543

544

Table 1. Individual social characteristics of the participants

Variable
Gender
Female
Male
Education level
Diploma of Nursing
Bachelor of Nursing
Bachelor of Psychology
Master of Nursing
Master of Psychology
Marital status
Married
Single


divorced

Age

Min- Max

Mean \pm Sd

Work experience (years)

Min- Max

Mean $\pm \mathrm{Sd}$

Min: Minimum; Max: Maximum; Sd: Standard

deviation

545

546

547

548

549

550

Table 2. Themes and categories extracted from content analysis

Theme

Category

Privacy

Physical-

sexual

privacy

Psychologic

al privacy

Informationa

l privacy

Possessive

privacy

Respecting individual identity

Respect for

age identity

Respect for

religious

identity

Avoiding

pity

behaviors 
Avoiding

discriminatio

n

Comprehensive support

Familial

support

Social

awareness

support

Financial

support

551

552 